\title{
Local Ischemic Post-Conditioning: Moving in the Right Direction?
}

\author{
Rajesh K. Kharbanda \\ Oxford Biomedical Research Centre, Department of Cardiology, Oxford University Hospitals, Oxford, UK
}

\section{Introduction}

Prognosis after ST-elevation acute coronary syndrome (STE-ACS) is driven by the degree of impact on left ventricular function secondary to myocardial injury [1]. In the early phase of STE-ACS, this can be manifest through the development of cardiogenic shock and subsequently through the development of chronic heart failure. Primary angioplasty (PPCI) has improved the effectiveness of reperfusion therapy. PPCI reduces the extent of myocardial injury and improves clinical outcomes after STEACS, but significant additional gains from further technical improvements alone are difficult to envisage. Increased understanding of 'reperfusion injury' and its demonstration in patients undergoing PPCI has focused attention upon adjuvant interventions at the time of PPCI that may further reduce myocardial injury (caused during and by reperfusion) and have a beneficial impact on outcomes [2].

\section{Local Ischemic Post-Conditioning}

Following the first descriptions of local ischemic postconditioning in animal models by $\mathrm{Na}$ et al. [3] and Zhao et al. [4], there was rapid translation into human clinical studies [5]. At this time, the change in clinical practice from thrombolysis to PPCI provided the ideal opportunity for direct investigation of local ischemic post-conditioning and to explore the clinical merits of the cardioprotective effects observed in animal models. Specifical- ly, methods were developed to use the angioplasty balloon within the infarct-related coronary artery to re-occlude blood flow and experimentally modify the early reperfusion phase. In this issue of Cardiology, Wei et al. [6] present a meta-analysis of 13 studies conducted between 2005 and 2011 involving a total of 673 patients, of which 352 were treated by local ischemic post-conditioning during PPCI. They report data relating to its effect on: (i) biochemical measures of infarct size, (ii) imaging measures of infarct size, (iii) indices of reperfusion (ST-segment resolution, blush grade and TIMI frame count), (iv) measures of left ventricular function and (v) clinical outcome measures [6]. They report a positive consistent effect of local ischemic post-conditioning on all of these measures, despite the varying protocols used in these studies. The reported 7.8\% (95\% CI 7.12-8.46) difference in LV ejection fraction at 3 months after the infarct is clinically meaningful, and there is a suggestion of reduced progression towards heart failure. Two other metaanalyses in this area strongly support these findings with regard to the effects on infarct size and LV ejection fraction $[7,8]$.

\section{Ready for Translation?}

The positive proof-of-principal studies conducted to date have understandably been in small and highly selected populations, comprising patients who mainly experience left anterior descending infarction, with TIMI 0 flow. The early translation of the technique meant that different

\section{KARGER}

Fax +4161306 1234 E-Mail karger@karger.ch www.karger.com

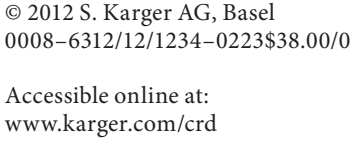

Dr. Rajesh K. Kharbanda

Oxford Biomedical Research Centre

Department of Cardiology, Oxford University Hospitals

Oxford OX3 9DU (UK)

E-Mail rajesh.kharbanda@ouh.nhs.uk 
protocols were tested in small numbers of patients. Most recent studies have adopted four 60 -second inflations; however, it is unclear whether this is the optimal timing for intervention. Furthermore, direct stenting appears to be an important factor influencing the achievement of positive clinical outcomes [8]. Sequential dilation with a pre-dilatation balloon may disrupt and embolise the thrombus, thus limiting myocardial salvage. In addition, no randomized studies have explored the effects of post-conditioning in the context of thrombectomy, which is now often considered standard of care [9]. Furthermore, the impact of comorbidities, such as diabetes, is not known.

Some recent studies, which were not eligible for inclusion in this report, have failed to demonstrate a significant benefit from post-conditioning and have highlighted confounding factors that may influence its efficacy [10]. Future studies, particularly those using MRI salvage as a measure of outcomes, should consider taking into account these factors which include age, gender and stenting technique, as well as the direct impact of post-conditioning on myocardial oedema (a key measure of the area at risk) [11]. The ongoing DANAMI-3 study has adequate size and power to show the effects of local post-conditioning on the clinical endpoints of death, re-infarction and heart failure and we can anticipate that it will provide a clearer understanding of the clinical relevance of this intervention.

\section{What about the Future?}

The clinical situation is often more complex than research; clinicians are faced with patients of varying ages, comorbidities and drug regimens, who may present early or very late in the course of their infarct and have differing infarct-related arterial flow or collateralization. Against this backdrop of heterogeneity, it is likely that multiple cardioprotective interventions, which target parallel and synergistic pathways, would be the most effective strategy. As we develop a greater understanding of the determinants of the efficacy of these cardioprotective conditioning techniques once they are translated into the clinical arena, we will be better placed to apply them in a personalized fashion to benefit patients.

The new meta-analysis from Wei et al. [6] presents us with data that clearly demonstrate the benefits of postconditioning in a highly selected group with important effects on limiting infarct size and optimising the 3-month ejection fraction, and therefore support ongoing clinical research to refine knowledge in this area. Whilst it would be premature to encourage wide application of this manoeuvre in unselected PPCI patients at this early stage of translation, the results from large ongoing clinical studies can be eagerly awaited.

\section{References}

1 Miller TD, Christian TF, Hopfenspirger MR, Hodge DO, Gersh BJ, Gibbons RJ: Infarct size after acute myocardial infarction measured by quantitative tomographic $99 \mathrm{mTc}$ sestamibi imaging predicts subsequent mortality. Circulation 1995;92:334-341.

-2 Schwartz Longacre L, Kloner RA, Arai AE, et al: New horizons in cardioprotection: recommendations from the 2010 National Heart, Lung, and Blood Institute Workshop. Circulation 2011;124:1172-1179.

- 3 Na HS, Kim YI, Yoon YW, Han HC, Nahm $\mathrm{SH}$, Hong SK: Ventricular premature beatdriven intermittent restoration of coronary blood flow reduces the incidence of reperfusion-induced ventricular fibrillation in a cat model of regional ischemia. Am Heart J 1996;132:78-83.
4 Zhao ZQ, Corvera JS, Halkos ME, et al: Inhibition of myocardial injury by ischemic postconditioning during reperfusion: comparison with ischemic preconditioning. Am J Physiol Heart Circ Physiol 2003;285:H579-H588.

-5 Staat P, Rioufol G, Piot C, et al: Postconditioning the human heart. Circulation 2005; 112:2143-2148.

6 Wei Y, Ruan L, Zhou G, Zhao L, Qi B, Ouyang P, Jin Z, Zhang C, Liu S: Local ischemic postconditioning during primary percutaneous coronary intervention: a meta-analysis. Cardiology 2012;123:225-233.

7 Hansen PR, Thibault H, Abdulla J: Postconditioning during primary percutaneous coronary intervention: a review and meta-analysis. Int J Cardiol 2010;144:22-25.

8 Zhou C, Yao Y, Zheng Z, et al: Stenting technique, gender, and age are associated with cardioprotection by ischaemic postconditioning in primary coronary intervention: a systematic review of 10 randomized trials. Eur Heart J 2012, E-pub ahead of print.
9 Steg PG, James SK, Atar D, et al: ESC Guidelines for the management of acute myocardial infarction in patients presenting with ST-segment elevation: The Task Force on the management of ST-segment elevation acute myocardial infarction of the European Society of Cardiology (ESC). Eur Heart J 2012, E-pub ahead of print.

10 Freixa X, Bellera N, Ortiz-Perez JT, et al: Ischaemic postconditioning revisited: lack of effects on infarct size following primary percutaneous coronary intervention. Eur Heart J 2012;33:103-112.

11 Thuny F, Lairez O, Roubille F, et al: Post-conditioning reduces infarct size and edema in patients with ST-segment elevation myocardial infarction. J Am Coll Cardiol 2012;59: 2175-2181. 